\title{
Robustness of superconducting properties to transition metal substitution and impurity phases in $\mathrm{Fe}_{1-x} \mathrm{~V}_{x} \mathrm{Se}$
}

\author{
Franziska K. K. Kirschner, ${ }^{1, *}$ Daniel N. Woodruff, ${ }^{2}$ Matthew J. Bristow, ${ }^{1}$ Franz Lang, ${ }^{1}$ Peter J. Baker, ${ }^{3}$ \\ Simon J. Clarke, ${ }^{2}$ and Stephen J. Blundell $\oplus^{1, \dagger}$ \\ ${ }^{1}$ Department of Physics, University of Oxford, Clarendon Laboratory, Parks Road, Oxford OX1 3PU, United Kingdom \\ ${ }^{2}$ Department of Chemistry, University of Oxford, Inorganic Chemistry Laboratory, South Parks Road, Oxford OX1 3QR, United Kingdom \\ ${ }^{3}$ ISIS Facility, STFC Rutherford Appleton Laboratory, Chilton, Didcot, Oxfordshire OX11 OQX, United Kingdom
}

(Received 3 June 2019; revised manuscript received 12 August 2019; published 23 September 2019)

\begin{abstract}
We have performed transverse- and zero-field muon spin rotation/relaxation experiments, as well as magnetometry measurements, on samples of $\mathrm{Fe}_{1-x} \mathrm{~V}_{x} \mathrm{Se}$ and their $\mathrm{Li}+\mathrm{NH}_{3}$ intercalates $\mathrm{Li}_{0.6}\left(\mathrm{NH}_{2}\right)_{0.2}\left(\mathrm{NH}_{3}\right)_{0.8}$ $\mathrm{Fe}_{1-x} \mathrm{~V}_{x}$ Se. We examine the low vanadium substitution regime: $x=0.005,0.01$, and 0.02 . The intercalation reaction significantly increases the critical temperature $\left(T_{\mathrm{c}}\right)$ and the superfluid stiffness for all $x$. The nonintercalated samples all exhibit $T_{\mathrm{c}} \approx 8.5 \mathrm{~K}$ while the intercalated samples all show an enhanced $T_{\mathrm{c}}>40 \mathrm{~K}$. Vanadium substitution has a negligible effect on $T_{\mathrm{c}}$, but seems to suppress the superfluid stiffness for the nonintercalated samples and weakly enhance it for the intercalated materials. The optimal substitution level for the intercalated samples is found to be $x=0.01$, with $T_{\mathrm{c}} \approx 41 \mathrm{~K}$ and $\lambda_{a b}(0) \approx 0.18 \mu \mathrm{m}$. The nonintercalated samples can be modeled with either a single $d$-wave superconducting gap or with an anisotropic gap function based on recent quasiparticle imaging experiments, whereas the intercalates display multigap nodal behavior which can be fitted using $s+d$ - or $d+d$-wave models. Magnetism, likely from iron impurities, appears after the intercalation reaction and coexists and competes with the superconductivity. However, it appears that the superconductivity is remarkably robust to the impurity phase, providing an avenue to stably improve the superconducting properties of transition metal substituted FeSe.
\end{abstract}

DOI: 10.1103/PhysRevB.100.094527

\section{INTRODUCTION}

The discovery of superconductivity in iron-based systems $[1,2]$ has produced a range of different high-temperature superconductors. Among these compounds are those based on $\mathrm{FeSe}$, which in its undoped form [3] has a critical temperature [4] $T_{\mathrm{c}} \approx 8 \mathrm{~K}$. Pressure [5], molecular intercalation [6], and thin-film fabrication [7] can significantly enhance the superconductivity in FeSe, with $T_{\mathrm{c}}$ reaching over $100 \mathrm{~K}$. The studies on FeSe intercalates in particular have revealed a remarkable robustness of the superconducting properties to structural disorder [8]. Another common method for chemically altering $\mathrm{FeSe}$, and consequently enhancing superconductivity, is through substitution on the chalcogenide site. Through tuning the substitution fraction $x$ in $\mathrm{FeSe}_{1-x} \mathrm{~S}_{x}$ and $\mathrm{FeSe}_{1-x} \mathrm{Te}_{x}, T_{\mathrm{c}}$ increases from the FeSe value by $20 \%$ and $75 \%$ respectively [9].

On the other hand, transition metal substitution of iron $\left(\mathrm{Fe}_{1-x} T_{x} \mathrm{Se}\right.$ for transition metal $\left.T\right)$ has had more mixed results. Superconductivity in $\mathrm{Fe}_{1-x} \mathrm{Cu}_{x} \mathrm{Se}$ is suppressed for $x>1.5 \%$, and $x>4 \%$ drives the sample through a metal-insulator transition [10]. This is thought to occur owing to $\mathrm{Cu}$ atoms disrupting the electronic structure, and eventually causing the metal-insulator transition due to Anderson localization [11]. Co and Ni substitutions have been found to either suppress [9]

\footnotetext{
*franziska.kirschner@physics.ox.ac.uk

${ }^{\dagger}$ stephen.blundell@physics.ox.ac.uk
}

$T_{\mathrm{c}}$ or destroy superconductivity completely [12]. For $T=$ $\mathrm{Mn}, \mathrm{V}, \mathrm{Cr}$, and $\mathrm{Ti}$, it has been found that $x$ can be tuned to optimize [12-14] $T_{\mathrm{c}}$. An optimum $T_{\mathrm{c}} \approx 11 \mathrm{~K}$ was found [12] for $\mathrm{Fe}_{0.98} \mathrm{~V}_{0.02} \mathrm{Se}$. It is thought that these highly elementdependent results arise from both the ionic size and level of impurity phases. Increased pressure on FeSe increases the fraction of hexagonal impurity phase in the sample, which at first increases $T_{\mathfrak{c}}$, and then rapidly suppresses it [5]. It has been theorized that the amount of hexagonal phase could vary as a function of chemical pressure which is related to the size of the substituted transition metal ions [12], although the effect of chemical pressure from chalcogenide substitution in $\mathrm{FeSe}_{1-x}(\mathrm{~S}, \mathrm{Te})_{x}$ appears to be inconsistent with hydrostatic pressure studies [9]. A study on transition metal substitution in $\mathrm{FeSe}_{0.5} \mathrm{Te}_{0.5}$ saw similar results to those for $\mathrm{FeSe}$ and suggested that the differing magnetic properties between the transition metal ions may induce different local impurity moments and net carrier concentrations [15]. As a result of this variation, it has also been suggested that the pairing symmetry of transition metal substituted compounds may not be pure $s$ or $d$ wave.

In this paper, we perform muon spin-relaxation and rotation $(\mu \mathrm{SR})$ and magnetometry experiments on three samples of $\mathrm{Fe}_{1-x} \mathrm{~V}_{x} \mathrm{Se}$ (with $x=0.005,0.01$, and 0.02) and their ammonia intercalates [chemical formula $\mathrm{Li}_{0.6}\left(\mathrm{NH}_{2}\right)_{0.2}$ $\left(\mathrm{NH}_{3}\right)_{0.8} \mathrm{Fe}_{1-x} \mathrm{~V}_{x} \mathrm{Se}$, labeled as $\left.x+\mathrm{NH}_{3}\right]$. Using transverse field (TF) $\mu \mathrm{SR}$, we extract the superconducting properties of all samples, and find that the superfluid stiffness and critical temperature both increase significantly after intercalation. We 
also observe superconductivity with an anisotropic gap in both classes of samples, with the opening of a second gap in the intercalates. Zero field (ZF) $\mu$ SR and magnetometry measurements reveal a strong magnetic signal in the intercalates, which is absent in the nonintercalated samples, likely arising from iron-based impurities. We find that the superconductivity remains robust, despite the introduction of very small amounts of elemental iron impurities produced during the intercalation reactions.

\section{EXPERIMENTAL DETAILS}

Synthesis of $\mathrm{Fe}_{1-x} \mathrm{M}_{x}$ Se. Iron powder (99.998\%, Alfa Aesar), selenium powder (99.999\%, Alfa Aesar) and vanadium powder (>99.99\%) were ground together in the desired stoichiometry in an agate mortar and pestle for $10 \mathrm{~min}$ before being sealed inside a silica ampoule. This was heated to $700{ }^{\circ} \mathrm{C}\left(2{ }^{\circ} \mathrm{C} / \mathrm{min}\right)$ and kept there for $48 \mathrm{~h}$ before being furnace cooled to room temperature. The grey powder was then reground and sealed inside a fresh silica ampoule. This was heated to $700{ }^{\circ} \mathrm{C}\left(2{ }^{\circ} \mathrm{C} / \mathrm{min}\right)$ for $36 \mathrm{~h}$ before being cooled to $400{ }^{\circ} \mathrm{C}$ and annealed for 10 days before being quenched to $0^{\circ} \mathrm{C}$. The isolated powders generally had small amounts ( $<5 \%$ by weight) of $\alpha$-FeSe impurity and were used as isolated for the various intercalation reactions performed in this study.

Synthesis of $\mathrm{Li}_{z}\left(\mathrm{NH}_{3}\right)_{y} \mathrm{Fe}_{1-x} \mathrm{~V}_{x} \mathrm{Se}$. A synthetic procedure was used similar to the one we have reported previously [6]. A sample of $\mathrm{Fe}_{1-x} \mathrm{~V}_{x} \mathrm{Se}(500 \mathrm{mg})$ was placed inside a Schlenk tube along with lithium metal $(\approx 13 \mathrm{mg})$ and a Teflon-coated magnetic stirrer bar. The Schlenk tube was evacuated and cooled to $-78^{\circ} \mathrm{C}$ using a $\mathrm{CO}_{2} /$ isopropanol bath. Ammonia $(\approx 15 \mathrm{ml})$ was condensed into the flask to afford a dark blue solution. This was stirred for $4 \mathrm{~h}$ at $-78^{\circ} \mathrm{C}$ before the flask was allowed to warm to room temperature naturally within the $\mathrm{CO}_{2}$ /isopropanol bath; all the ammonia evaporated via a mercury bubbler. Once at room temperature the flask was placed under dynamic vacuum for 2 min before the dark grey material was then isolated inside an argon-filled glovebox. The intercalated samples exhibit sensitivity to air (see the Appendix) and precautions were taken to avoid exposure to air for these samples in subsequent characterization experiments.

Diffraction measurements. X-ray powder diffraction (XRPD) measurements were performed on instrument I11 [16] at the Diamond Light Source, with 0.826- $\AA$ x rays and the position sensitive (MYTHEN) detector. Rietveld refinements against powder-diffraction data were conducted using the TOPAS Academic software [17].

Magnetometry. Field-cooled (FC) and zero-field-cooled (ZFC) magnetometry measurements were made using a Quantum Design MPMS superconducting quantum interference device magnetometer which utilized measuring fields of 2050 Oe in order to characterize the superconducting state and up to $7 \mathrm{~T}$ to probe the normal-state susceptibilities. Samples were sequestered from air in gelatin capsules. Susceptibilities were corrected for the effect of demagnetizing fields arising from the shape of the sample.

Muon spin-relaxation measurements. $\mu \mathrm{SR}$ experiments [18,19] were performed using a ${ }^{3} \mathrm{He}$ cryostat mounted on the MuSR spectrometer at the ISIS pulsed muon facility (Rutherford Appleton Laboratory, UK) [20]. TF measurements, in which an external magnetic field is applied transverse to the initial muon spin polarization, were made to identify the superconducting ground state and its evolution with $x$. ZF measurements were carried out on the $x=0.02+\mathrm{NH}_{3}$ sample in order to test for magnetic phases in the sample. All of the $\mu \mathrm{SR}$ data were analyzed using WiMDA [21].

\section{SUPERCONDUCTIVITY}

To determine the superconducting properties, all samples were measured in a transverse field of $B_{0}=15 \mathrm{mT}$ at temperatures $T$ above and below $T_{\mathrm{c}}$. Sample spectra for $x=0.01$, as plotted in Fig. 1(a), show a clear increase in relaxation in the superconducting state (compared to the normal state), arising from the inhomogeneous magnetic field distribution of the vortex lattice. The data were fitted with the two-component function

$$
\begin{aligned}
A(t)= & A_{B} \cos \left(\gamma_{\mu} B_{0} t+\phi\right) e^{-\lambda_{\mathrm{TF}} t} \\
& +A_{\mathrm{SC}} \cos \left(\gamma_{\mu} B_{\mathrm{SC}} t+\phi\right) e^{-\sigma^{2} t^{2} / 2},
\end{aligned}
$$

where $\gamma_{\mu}=2 \pi \times 135.5 \mathrm{MHzT}^{-1}$ is the gyromagnetic ratio of the muon and $\phi$ is a phase related to the detector geometry, with $\phi$ fitted for each of the eight detector groups. The first term represents those muons which are not in the superconducting volume and precess only in the external magnetic field. These muons experience a small Lorentzian relaxation due to magnetism in the sample (see below for further discussion), with $\lambda_{\mathrm{TF}} \approx 0.1-0.2 \mu \mathrm{s}^{-1}$ showing little variation between samples. The second term arises from muons in the superconducting volume, which experience a Gaussian broadening $\sigma(T)=\sqrt{\sigma_{\mathrm{SC}}^{2}(T)+\sigma_{\text {nucl }}^{2}}$. This broadening consists of a temperature-dependent component from the vortex lattice, and a temperature-independent component from static nuclear moments [plotted in Fig. 1(b)]. $\sigma_{\text {nucl }}$ is much higher for the intercalated samples, compared to the nonintercalated samples which may reflect a contribution from static nonnuclear (i.e., electronic) moments, although we note that this contribution is temperature independent.

The field shifts caused by the vortex lattice $\Delta B=B_{\mathrm{SC}}-$ $B_{0}$ in the nonintercalated and intercalated samples are shown in Figs. 1(c) and 1(d), respectively. There is a clear negative shift in the peak field as the samples transition into their superconducting states; this is a characteristic feature of the vortex lattice [22].

In order to extract the penetration depth from $\sigma_{\mathrm{SC}}$, a conversion [23]

$$
\sigma_{\mathrm{SC}}=0.0609 \gamma_{\mu} \phi_{0} \lambda_{\text {eff }}^{-2}(T)
$$

was used; $\phi_{0}=2.069 \times 10^{-15} \mathrm{~Wb}$ is the magnetic-flux quantum. All of the samples were anisotropic and polycrystalline and so it can therefore be assumed that the effective penetration depth $\lambda_{\text {eff }}$ is dominated by the in-plane penetration depth $\lambda_{a b}$, and so [24] $\lambda_{\text {eff }}=3^{1 / 4} \lambda_{a b}$. The temperature dependencies of $\lambda_{a b}^{-2}$ for the nonintercalated and intercalated compounds are plotted in Figs. 1(e) and 1(f), respectively.

The data in Figs. 1(e) and 1(f) have been fitted with singleand two-gap BCS models involving $s$ - and $d$-wave gaps. 

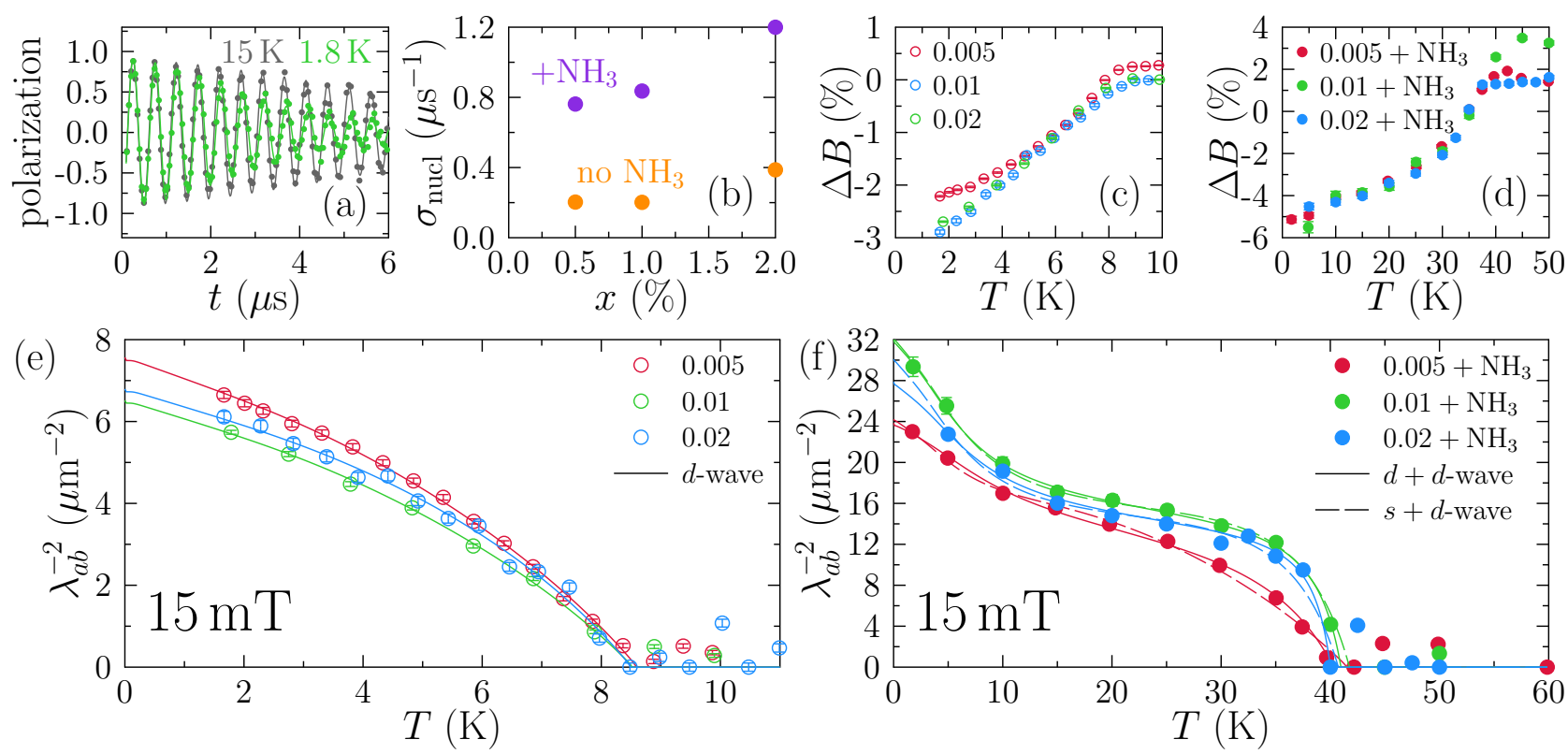

FIG. 1. (a) Sample TF- $\mu$ SR spectra above and below $T_{\mathrm{c}}$ for $x=0.01$. Fits as in Eq. (1) are also plotted. (b) Dependence of the nuclear contribution to the superconducting relaxation [described in Eq. (1)] on vanadium substitution for both intercalated $\left(+\mathrm{NH}_{3}\right)$ and nonintercalated $\left(\right.$ no $+\mathrm{NH}_{3}$ ) samples. The temperature dependence of the field width of the superconducting vortex lattice is given in (c) and (d) for the nonintercalated and intercalated samples respectively. The temperature dependence of the inverse square penetration depth for nonintercalated and intercalated samples is shown in (e) and (f) respectively. The data in (e) have been fitted with a single-gap $d$-wave function, and the data in (f) have been fitted with two-gap $d+d$ and $s+d$ models.

The BCS model of the normalized superfluid density of a superconductor is given by [25]

$$
\begin{aligned}
\tilde{n}_{\mathrm{s}}(T) & =\frac{\lambda_{a b}^{-2}(T)}{\lambda_{a b}^{-2}(0)} \\
& =1+\frac{1}{\pi} \int_{0}^{2 \pi} \int_{\Delta(\phi, T)}^{\infty} \frac{\partial f}{\partial E} \frac{E d E d \phi}{\sqrt{E^{2}-\Delta^{2}(\phi, T)}},
\end{aligned}
$$

where $\Delta(\phi, T)$ is the superconducting gap function, and $f=\left[1+\exp \left(E / k_{\mathrm{B}} T\right)\right]^{-1}$ is the Fermi function. The gap function can be approximated as $\Delta(\phi, T)=$ $\Delta(\phi) \tanh \left\{1.82\left[1.018\left(T_{\mathrm{c}} / T-1\right)\right]^{0.51}\right\}$. The angular gap function $\Delta(\phi)=\Delta_{0}$ for $s$-wave superconductors and $\Delta(\phi)=\Delta_{0} \cos (2 \phi)$ for $d$-wave (nodal) superconductors. Multigap systems can be represented by a sum of the $\tilde{n}_{\mathrm{s}}(0)$ values for each individual gap, weighted by a factor $w$ using

$$
\tilde{n}_{\mathrm{s}}(T)=w \tilde{n}_{\mathrm{s}}^{(\text {gap 1) }}(T)+(1-w) \tilde{n}_{\mathrm{s}}^{(\text {gap 2) }}(T) .
$$

After trying combinations of $s$-wave and $d$-wave gap functions using Eq. (4). We find that the nonintercalated samples are best described by a single-gap $d$-wave model (though we note the sensitivity [26] of the gap to disorder in FeSe) and the extracted gap values (in the range 1-2 meV) are consistent with those found for pure FeSe using other techniques [27-29]. After intercalation, an additional gap opens up: the intercalated samples are described well by either $s+d$ or $d+d$ gaps (these two models gave very similar $\chi^{2}$ values). The superconducting parameters associated with the best fits are given in Table I. On the surface of pure FeSe, two gaps have been measured using quasiparticle interference

\begin{tabular}{|c|c|c|c|c|c|c|c|c|c|}
\hline Sample & $\begin{array}{c}a \\
(\AA)\end{array}$ & $\begin{array}{c}c \\
(\AA)\end{array}$ & $c / a$ & Gap & $\begin{array}{c}T_{\mathrm{c}} \\
(\mathrm{K})\end{array}$ & $\begin{array}{c}\Delta_{1}(\text { symmetry }) \\
(\mathrm{meV})\end{array}$ & $\begin{array}{c}\Delta_{2} \text { (symmetry) } \\
(\mathrm{meV})\end{array}$ & $w$ & $\begin{array}{c}\lambda_{a b} \\
(\mu \mathrm{m})\end{array}$ \\
\hline 0.005 & $3.77076(3)$ & $5.52137(4)$ & 1.4643 & $d$ & $8.6(1)$ & $1.72(8)(d)$ & & & $0.36(1)$ \\
\hline 0.01 & $3.77129(2)$ & $5.52105(3)$ & 1.4640 & $d$ & $8.5(3)$ & $1.71(15)(d)$ & & & $0.39(1)$ \\
\hline 0.02 & $3.77152(3)$ & $5.52164(6)$ & 1.4640 & $d$ & $8.5(1)$ & $1.83(14)(d)$ & & & $0.38(1)$ \\
\hline \multirow[t]{2}{*}{$0.005+\mathrm{NH}_{3}$} & \multirow[t]{2}{*}{$3.8315(1)$} & \multirow[t]{2}{*}{$16.3968(7)$} & \multirow[t]{2}{*}{4.2795} & $s+d$ & $41.1(8)$ & $0.62(1)(s)$ & $0.14(1)(d)$ & $0.61(9)$ & $0.20(2)$ \\
\hline & & & & $d+d$ & $40.2(9)$ & $1.1(1)(d)$ & $0.18(1)(d)$ & $0.64(11)$ & $0.19(2)$ \\
\hline \multirow{2}{*}{$0.01+\mathrm{NH}_{3}$} & \multirow[t]{2}{*}{$3.8336(1)$} & \multirow[t]{2}{*}{$16.3429(4)$} & \multirow[t]{2}{*}{4.2631} & $s+d$ & $41.7(6)$ & $1.23(5)(s)$ & $0.14(1)(d)$ & $0.45(5)$ & $0.18(1)$ \\
\hline & & & & $d+d$ & $40.9(1)$ & $2.1(1)(d)$ & $0.16(1)(d)$ & $0.53(4)$ & $0.18(1)$ \\
\hline \multirow[t]{2}{*}{$0.02+\mathrm{NH}_{3}$} & \multirow[t]{2}{*}{$3.8295(1)$} & \multirow[t]{2}{*}{$16.4504(6)$} & \multirow[t]{2}{*}{4.2957} & $s+d$ & $40.7(4)$ & $1.17(1)(s)$ & $0.14(1)(d)$ & $0.46(9)$ & $0.19(2)$ \\
\hline & & & & $d+d$ & $40.0(1)$ & $2.3(1)(d)$ & $0.16(1)(d)$ & $0.55(9)$ & $0.20(2)$ \\
\hline
\end{tabular}
imaging [29,30] and their angular dependence mapped out

TABLE I. Fitted parameters for the temperature dependence of $\lambda_{a b}^{-2}$ [plotted in Figs. 1(e) and 1(f)], using the fit in Eq. (3). 


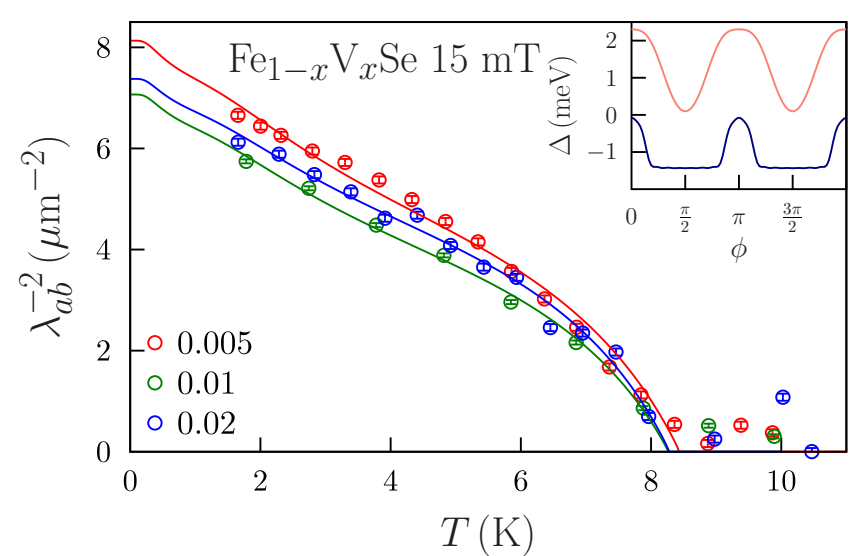

FIG. 2. Fits of the data for $\mathrm{Fe}_{1-x} \mathrm{~V}_{x}$ Se to a gap function (see inset) based on the results of quasiparticle imaging experiments $[29,30]$ on the surface of bulk FeSe, as described in the main text.

(see Fig. 2). We have used these measured gap functions and Eq. (4) to fit the data on our V-substituted FeSe samples and achieve a reasonable agreement with the data (Fig. 2) and a very slightly lower estimate of the penetration depth $\left[\lambda_{a b}(0)=0.35(1), 0.37(1)\right.$, and $0.38(1) \mu \mathrm{m}$ for $x=0.005$, 0.01 , and 0.02 respectively]. For the intercalated samples, a fit using the measured gap [31] for monolayer FeSe was not successful (not shown). This is likely due to monolayer FeSe having a single Fermi-surface pocket [32-34] [as does $(\mathrm{Li}, \mathrm{Fe}) \mathrm{OHFeSe}$ [ [35] leading to a single gap with a more conventional temperature dependence [36]. Although our $\mu$ SR data cannot help us pin down the pairing symmetry precisely, it nevertheless provides strong evidence for two distinct gaps for the ammonia-intercalated materials, probably resulting from the additional pockets predicted for these compounds [37].

For the intercalated samples, we remark that the optimal substitution level $x=0.01$ gives the largest value of $T_{\mathrm{c}}$ and the shortest penetration depth [and therefore the largest superfluid stiffness, which is proportional to $\left.\lambda_{a b}^{-2}(0)\right]$ though the variation in both parameters as a function of $x$ is very slight. It has previously been reported that optimum values of $x$ in transition metal doped FeSe exist [12], above which $T_{\mathrm{c}}$ decreases, although for $\mathrm{V}$ substitution, a previous study on nonintercalated samples of $\mathrm{Fe}_{1-x} \mathrm{~V}_{x} \mathrm{Se}$ found the optimal point to be $x=0.02$ [12]. For our nonintercalated samples, the superconducting properties exhibit very little $x$ dependence (and detailed $\mu$ SR studies have been performed in pure FeSe $[38,39])$. We find, however, that transition metal substitution significantly decreases the penetration depth, compared to the undoped case $\left[\lambda_{a b}(0) \approx 0.41 \mu \mathrm{m}\right.$ and $\approx 0.25 \mu \mathrm{m}$ for the nonintercalated and intercalated FeSe samples respectively].

\section{MAGNETISM}

To examine the relaxation due to magnetism in the TF- $\mu$ SR data, FC and ZFC bulk magnetization measurements were carried out, and are plotted in Fig. 3(a). The nonintercalated samples were found to undergo a superconducting transition at the expected temperatures, with no strong magnetic signal above $T_{\mathrm{c}}$. However for the intercalated samples, we observe
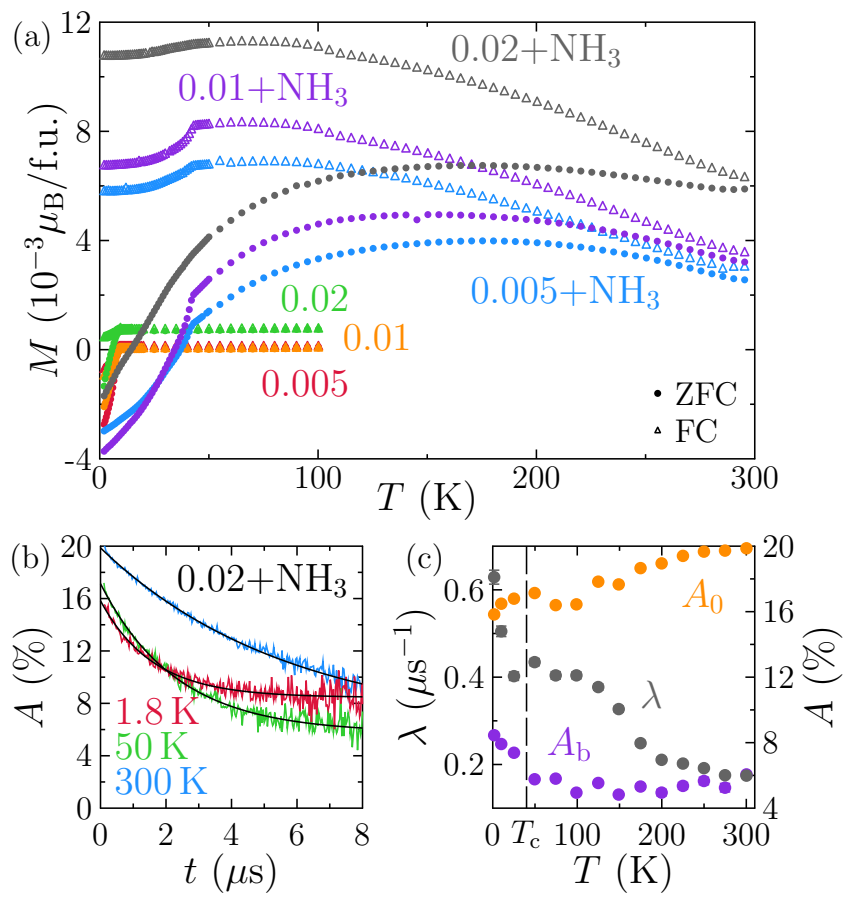

FIG. 3. (a) Temperature dependence of the magnetization for all samples, measured in Bohr magnetons per formula unit. (b) ZF$\mu \mathrm{SR}$ asymmetry for $x=0.02+\mathrm{NH}_{3}$ at a range of temperatures. The black lines give fits as in Eq. (5). (c) Temperature dependence of relaxation $\lambda$, and initial and baseline asymmetries $\left(A_{0}\right.$ and $A_{\mathrm{b}}$ respectively) of the $\mathrm{ZF}-\mu \mathrm{SR}$ asymmetry for $x=0.02+\mathrm{NH}_{3}$.

a clear enhancement in the magnetization, which appears to arise from elemental iron impurities. This enhancement overrides any superconducting signal and, for $x=0.01+\mathrm{NH}_{3}$ and $x=0.02+\mathrm{NH}_{3}$, the susceptibility is positive even well below $T_{\mathrm{c}}$. From this we can conclude that the superconductivity observed in the TF- $\mu$ SR is likely strongly localized in a small volume fraction

We performed ZF- $\mu$ SR measurements on the $x=0.02+$ $\mathrm{NH}_{3}$ sample, to further investigate the bulk magnetic signal observed in the magnetization data. Sample spectra well below, near, and above $T_{\mathrm{c}}$ are plotted in Fig. 3(b). There appears to be no Kubo-Toyabe relaxation, indicating that the magnetism is likely from electronic moments rather than nuclear moments. The data were well modeled with a singlecomponent Lorentzian relaxation:

$$
A(t)=\left(A_{0}-A_{\mathrm{b}}\right) e^{-\lambda t}+A_{\mathrm{b}},
$$

where $A_{0}$ and $A_{\mathrm{b}}$ are the initial and baseline asymmetries respectively, and $\lambda$ is the relaxation rate. The fitted values of $A_{0}, A_{\mathrm{b}}$, and $\lambda$ are plotted in Fig. 3(c).

As $T$ decreases, we find the initial asymmetry to decrease. This is indicative of a fast-relaxing phase in the sample, which is outside the resolution of the spectrometer. An increase in the baseline asymmetry at low $T$ suggests a higher fraction of muons landing in areas of the sample with no magnetic field (we note that $A_{\mathrm{b}}$ also contains a contribution from muons in the sample holder and cryostat, but this contribution is expected to be temperature independent). One possible explanation of this behavior is that the relaxation arises from 
magnetic puddles containing iron impurities that freeze-out at low temperatures to create areas of static spin distributions with high resultant dipolar fields. This change in the asymmetries and interpretation is consistent with the increase in $\lambda$ in Fig. 3(c). There are no oscillations in the spectra in Fig. 3(b), ruling out long-range magnetic order. We also find an increase in the magnitude of the gradient of $A_{0}, A_{\mathrm{b}}$, and $\lambda$ below $\approx T_{\mathrm{c}}$, which suggests the magnetism coexists and competes with the superconductivity. Despite the strongly magnetic phase, it appears that the superconductivity is robust to magnetism. There is evidence that superconductivity in FeSe can be strongly affected by the presence of disorder [40] but our results show that the presence of vanadium at low substitution levels produces insufficient disorder to have a marked effect on $T_{\mathrm{c}}$.

\section{CONCLUSION}

We have performed TF- and ZF- $\mu$ SR experiments, as well as magnetization measurements, on three samples of $\mathrm{Fe}_{1-x} \mathrm{~V}_{x} \mathrm{Se}$ and their ammonia intercalates. In contrast with a previous study [12], we find that the optimal value of $x=0.01$ gives the highest critical temperature and superfluid stiffness, although the dependence on $x$ is weak. Another study [41] has claimed that when the amount of additional $\mathrm{Fe}$ in interstitial sites between the FeSe layers reaches more than about 3\%, superconductivity can be destroyed. There were no measurable interstitial $\mathrm{Fe}$ ions in the $\mathrm{Fe}_{1-x} \mathrm{~V}_{x}$ Se phases according to the $\mathrm{x}$-ray-diffraction measurements, consistent with the presence of superconductivity (refined occupancy of $<1 \%$ with an uncertainty of $\sim 1 \%$ ). Intercalation increases these superconducting parameters significantly, similar to that seen in pure FeSe and its intercalate [6]. The nonintercalated samples all exhibit $T_{\mathrm{c}} \approx 8.5 \mathrm{~K}$ while the intercalated samples all show an enhanced $T_{\mathrm{c}}>40 \mathrm{~K}$. Vanadium substitution has a negligible effect on $T_{\mathrm{c}}$ but seems to suppress the superfluid stiffness for the nonintercalated samples but enhance it for the intercalated materials. The nonintercalated samples can be modeled with either a single $d$-wave superconducting gap or with an anisotropic gap function based on recent quasiparticle imaging experiments, whereas the intercalates display multigap nodal behavior which is best described using either $s+d$ or $d+d$-wave models. In the intercalation reactions with reducing sources of $\mathrm{Li}$, the thermodynamic products are $\mathrm{Li}_{2} \mathrm{Se}$ and elemental $\mathrm{Fe}$. In the reactions with $\mathrm{Li} / \mathrm{NH}_{3}$ to obtain the products reported here, the intercalates are metastable intermediates. As the susceptibility data show, some elemental $\mathrm{Fe}$ is formed by partial decomposition at about the $5 \%$ level according to the magnetization isotherms, but this does not destroy the superconductivity in the intercalate phase. The $\mathrm{ZF}-\mu \mathrm{SR}$ experiments suggest these impurities form localized magnetic regions, which coexist and compete with the superconducting phase. In Ref. [42] we found that in some samples the superconducting state coexisted with particles of expelled $\mathrm{Fe}$, and here we also find superconductivity is robust to the impurity phase. This suggests that the line nodes in the intercalates are likely symmetry imposed and the impurity phase does not induce fully gapped behavior. An important drawback for the intercalated materials however is that they are air sensitive (see the Appendix). Our results provide a route for creating intercalated FeSe compounds through transition metal substitution on the Fe site.

\section{ACKNOWLEDGMENTS}

We thank C. V. Topping for useful discussions. F.K.K.K. thanks Lincoln College, Oxford, for a doctoral studentship. Part of this work was performed at the Science and Technology Facilities Council (STFC) ISIS Facility, Rutherford Appleton Laboratory and the Diamond Light Source (Beamtime Allocation No. EE13284 and No. EE18786). We acknowledge funding from EPSRC under Grants No. EP/M020517/1 and No. EP/N023803/1, Oxford's Centre for Applied Superconductivity (CfAS), and the Leverhulme Trust under Grant No. RPG-2014-221.

\section{APPENDIX: AIR SENSITIVITY}

The intercalated samples were stored under argon before each $\mu \mathrm{SR}$ measurement and did not receive air exposure before being removed from the muon spectrometer. Following the $\mu$ SR measurements, the samples were kept wrapped in silver foil but were exposed to air for several weeks. They were then ground with glass (approximate 50:50 volume ratio) to avoid excessive absorption and preferred orientation problems and packed into $0.5-\mathrm{mm}$-diameter borosilicate capillaries. The samples were then measured using powder $\mathrm{x}$-ray diffraction at room temperature using the Mythen position sensitive detector at the I11 beamline (Diamond, UK). A comparison of the powder-diffraction patterns for the $x=0.005$ sample taken before and after the $\mu$ SR measurement (i.e., before and after air exposure) is shown in Fig. 4. Significant amounts of impurity phases (indicated by asterisks in Fig. 4) have formed from aerial decomposition of the product. However some of the intercalate remains. This experiment was repeated for other compositions and in some cases full sample decomposition had occurred, although we could not ensure that each sample had received precisely the same amount of exposure to air. In any case, these results serve to demonstrate that intercalated samples can suffer partial and potentially full degradation when exposed to air for at least several days. In contrast, the nonintercalated samples are stable in air.

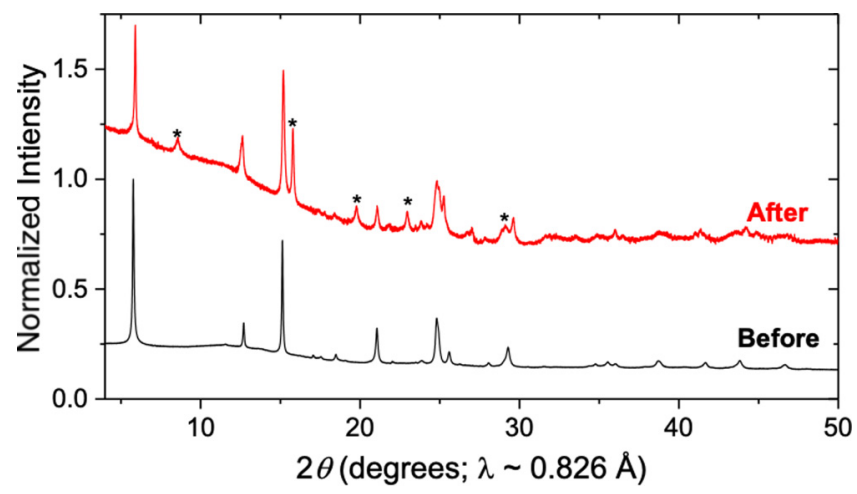

FIG. 4. Powder-diffraction data for the $x=0.005$ sample before and after exposure to air. The asterisks indicate new impurity peaks. Notice also the increase in the diffuse background. 
[1] Y. Kamihara, H. Hiramatsu, M. Hirano, R. Kawamura, H. Yanagi, T. Kamiya, and H. Hosono, J. Am. Chem. Soc. 128, 10012 (2006).

[2] Y. Kamihara, T. Watanabe, M. Hirano, and H. Hosono, J. Am. Chem. Soc. 130, 3296 (2008).

[3] A. I. Coldea and M. D. Watson, Annu. Rev. Condens. Matter Phys. 9, 125 (2018).

[4] F.-C. Hsu, J.-Y. Luo, K.-W. Yeh, T.-K. Chen, T.-W. Huang, P. M. Wu, Y.-C. Lee, Y.-L. Huang, Y.-Y. Chu, D.-C. Yan, and M.-K. Wu, Proc. Natl. Acad. Sci. U.S.A. 105, 14262 (2008).

[5] S. Medvedev, T. M. McQueen, I. A. Troyan, T. Palasyuk, M. I. Eremets, R. J. Cava, S. Naghavi, F. Casper, V. Ksenofontov, G. Wortmann, and C. Felser, Nat. Mater. 8, 630 (2009).

[6] M. Burrard-Lucas, D. G. Free, S. J. Sedlmaier, J. D. Wright, S. J. Cassidy, Y. Hara, A. J. Corkett, T. Lancaster, P. J. Baker, S. J. Blundell, and S. J. Clarke, Nat. Mater. 12, 15 (2013).

[7] J.-F. Ge, Z.-L. Liu, C. Liu, C.-L. Gao, D. Qian, Q.-K. Xue, Y. Liu, and J.-F. Jia, Nat. Mater. 14, 285 (2015).

[8] F. R. Foronda, S. Ghannadzadeh, S. J. Sedlmaier, J. D. Wright, K. Burns, S. J. Cassidy, P. A. Goddard, T. Lancaster, S. J. Clarke, and S. J. Blundell, Phys. Rev. B 92, 134517 (2015).

[9] Y. Mizuguchi, F. Tomioka, S. Tsuda, T. Yamaguchi, and Y. Takano, J. Phys. Soc. Jpn. 78, 074712 (2009).

[10] A. J. Williams, T. M. McQueen, V. Ksenofontov, C. Felser, and R. J. Cava, J. Phys.: Condens. Matter 21, 305701 (2009).

[11] S. Chadov, D. Schärf, G. H. Fecher, C. Felser, L. Zhang, and D. J. Singh, Phys. Rev. B 81, 104523 (2010).

[12] A. K. Yadav, A. V. Sanchela, A. D. Thakur, and C. V. Tomy, Solid State Commun. 202, 8 (2015).

[13] A. K. Yadav, A. D. Thakur, and C. V. Tomy, Solid State Commun. 151, 557 (2011).

[14] A. K. Yadav, A. D. Thakur, and C. V. Tomy, Phys. Rev. B 87, 174524 (2013).

[15] A. M. Zhang, T. L. Xia, L. R. Kong, J. H. Xiao, and Q. M. Zhang, J. Phys.: Condens. Matter 22, 245701 (2010).

[16] S. P. Thompson, J. E. Parker, J. Potter, T. P. Hill, A. Birt, T. M. Cobb, F. Yuan, and C. C. Tang, Rev. Sci. Instrum. 80, 075107 (2009).

[17] A. A. Coelho, TOPAS Academic Version 5 (Coelho Software, Brisbane, Australia, 2012).

[18] S. J. Blundell, Contemp. Phys. 40, 175 (1999).

[19] A. A. Yaouanc and P. D. de. Reotier, Muon Spin Rotation, Relaxation, and Resonance: Applications to Condensed Matter (Oxford University Press, Oxford, 2011).

[20] P. J. C. King, R. de Renzi, S. P. Cottrell, A. D. Hillier, and S. F. J. Cox, Phys. Scr. 88, 068502 (2013).

[21] F. L. Pratt, Physica B (Amsterdam) 289, 710 (2000).

[22] E. H. Brandt, Phys. Rev. B 37, 2349 (1988).

[23] E. H. Brandt, Phys. Rev. B 68, 054506 (2003).

[24] V. I. Fesenko, V. N. Gorbunov, and V. P. Smilga, Physica C (Amsterdam) 176, 551 (1991).

[25] B. S. Chandrasekhar and D. Einzel, Ann. Phys. (Berl.) 505, 535 (1993).
[26] Y. Sun, S. Kittaka, S. Nakamura, T. Sakakibara, P. Zhang, S. Shin, K. Irie, T. Nomoto, K. Machida, J. Chen, and T. Tamegai, Phys. Rev. B 98, 064505 (2018).

[27] C.-L. Song, Y.-L. Wang, P. Cheng, Y.-P. Jiang, W. Li, T. Zhang, Z. Li, K. He, L. Wang, J.-F. Jia, H.-H. Hung, C. Wu, X. Ma, X. Chen, and Q.-K. Xue, Science 332, 1410 (2011).

[28] S. Kasahara, T. Watashige, T. Hanaguri, Y. Kohsaka, T. Yamashita, Y. Shimoyama, Y. Mizukami, R. Endo, H. Ikeda, K. Aoyama, T. Terashima, S. Uji, T. Wolf, H. von Löhneysen, T. Shibauchi, and Y. Matsuda, Proc. Natl. Acad. Sci. USA 111, 16309 (2014).

[29] P. O. Sprau, A. Kostin, A. Kreisel, A. E. Böhmer, V. Taufour, P. C. Canfield, S. Mukherjee, P. J. Hirschfeld, B. M. Andersen, and J. C. S. Davis, Science 357, 75 (2017).

[30] A. Kreisel, B. M. Andersen, P. O. Sprau, A. Kostin, J. C. Séamus Davis, and P. J. Hirschfeld, Phys. Rev. B 95, 174504 (2017).

[31] Y. Zhang, J. J. Lee, R. G. Moore, W. Li, M. Yi, M. Hashimoto, D. H. Lu, T. P. Devereaux, D.-H. Lee, and Z.-X. Shen, Phys. Rev. Lett. 117, 117001 (2016).

[32] D. Liu, W. Zhang, D. Mou, J. He, Y.-B. Ou, Q.-Y. Wang, Z. Li, L. Wang, L. Zhao, S. He, Y. Peng, X. Liu, C. Chen, L. Yu, G. Liu, X. Dong, J. Zhang, C. Chen, Z. Xu, J. Hu, X. Chen, X. Ma, Q. Xue, and X. J. Zhou, Nat. Commun. 3, 931 (2012).

[33] Q.-Y. Wang, Z. Li, W.-H. Zhang, Z.-C. Zhang, J.-S. Zhang, W. Li, H. Ding, Y.-B. Ou, P. Deng, K. Chang, J. Wen, C.-L. Song, K. He, J.-F. Jia, S.-H. Ji, Y.-Y. Wang, L.-L. Wang, X. Chen, X.-C. Ma, and Q.-K. Xue, Chin. Phys. Lett. 29, 037402 (2012).

[34] S. N. Rebec, T. Jia, C. Zhang, M. Hashimoto, D.-H. Lu, R. G. Moore, and Z.-X. Shen, Phys. Rev. Lett. 118, 067002 (2017).

[35] L. Zhao, A. Liang, D. Yuan, Y. Hu, D. Liu, J. Huang, S. He, B. Shen, Y. Xu, X. Liu, L. Yu, G. Liu, H. Zhou, Y. Huang, X. Dong, F. Zhou, K. Liu, Z. Lu, Z. Zhao, C. Chen, Z. Xu, and X. J. Zhou, Nat. Commun. 7, 10608 (2016).

[36] P. K. Biswas, Z. Salman, Q. Song, R. Peng, J. Zhang, L. Shu, D. L. Feng, T. Prokscha, and E. Morenzoni, Phys. Rev. B 97, 174509 (2018).

[37] D. Guterding, H. O. Jeschke, P. J. Hirschfeld, and R. Valentí, Phys. Rev. B 91, 041112(R) (2015).

[38] R. Khasanov, K. Conder, E. Pomjakushina, A. Amato, C. Baines, Z. Bukowski, J. Karpinski, S. Katrych, H.-H. Klauss, H. Luetkens, A. Shengelaya, and N. D. Zhigadlo, Phys. Rev. B 78, 220510(R) (2008).

[39] P. K. Biswas, A. Kreisel, Q. Wang, D. T. Adroja, A. D. Hillier, J. Zhao, R. Khasanov, J.-C. Orain, A. Amato, and E. Morenzoni, Phys. Rev. B 98, 180501(R) (2018).

[40] A. E. Böhmer, V. Taufour, W. E. Straszheim, T. Wolf, and P. C. Canfield, Phys. Rev. B 94, 024526 (2016).

[41] T. M. McQueen, Q. Huang, V. Ksenofontov, C. Felser, Q. Xu, H. Zandbergen, Y. S. Hor, J. Allred, A. J. Williams, D. Qu, J. Checkelsky, N. P. Ong, and R. J. Cava, Phys. Rev. B 79, 014522 (2009).

[42] D. N. Woodruff, F. Schild, C. V. Topping, S. J. Cassidy, J. N. Blandy, S. J. Blundell, A. L. Thompson, and S. J. Clarke, Inorg. Chem. 55, 9886 (2016). 